\title{
Construction of a high-density genetic map: genotyping by sequencing (GBS) to map purple seed coat color (PsC) in hulless barley
}

Xiaohua Yao ${ }^{1,2,3,4}$, Kunlun Wu $\mathbf{u}^{1,2,3,4}$, Youhua Yao ${ }^{1,2,3,4}$, Yixiong Bai ${ }^{1,2,3,4}$, Jingxiu Ye ${ }^{1,2}$ and Dezhao Chi $i^{1,2,3,4^{*}}$ (D)

\begin{abstract}
Background: Colored hulless barley are more suitable in food processing compared to normal (yellow) varieties because it is rich in bioactive compounds and produces higher extraction pearling fractions. Therefore, seed coat color is an important agronomic trait for the breeding and study of hulless barley.

Results: Genotyping-by-sequencing single-nucleotide polymorphism (GBS-SNP) analysis of a doubled haploid (DH) mapping population (Nierumuzha $\times$ Kunlun10) was conducted to map the purple seed coat color genes (PSC). A high-density genetic map of hulless barley was constructed, which contains 3662 efficient SNP markers with 1129 bin markers. Seven linkage groups were resolved, which had a total length of $645.56 \mathrm{cM}$. Chromosome length ranged from $60.21 \mathrm{cM}$ to $127.21 \mathrm{cM}$, with average marker density of $0.57 \mathrm{cM}$. A total of five loci accounting for $3.79 \%$ to $23.86 \%$ of the observed phenotypic variation for PSC were detected using this high-density map. Five structural candidate genes (F3'M, HID, UF3GT, UFGT and 5MAT) and one regulatory factor (Ant1) related to flavonoid or anthocyanin biosynthesis were identified.

Conclusions: Five structural candidate genes and one regulatory factor related to flavonoid or anthocyanin biosynthesis have been identified using a high-density genetic map of hulless barley. This study lays the foundation for map-based cloning of PSC but provides a valuable tool for studying marker-trait associations and its application to marker-assisted breeding of hulless barley.
\end{abstract}

Keywords: Hulless barley-genotyping-by-sequencing (GBS), Purple seed coat color (Psc), Linkage analysis

\section{Background}

Hulless barley (Hordeum vulgare L. var. nudum Hook. f.) is a self-reproducing annual species that produces naked grains. Hulless barley is widely grown on the QinghaiTibet Plateau. It has served as a staple food for the Tibetan people since the fifth century CE [1]. The seed coat color is an important agronomic trait in crops due to its association with unique biological activity and function in healthcare [2]. Upon maturity, barley grains may display different pigmentations. Barley has a variety of seed coat

\footnotetext{
*Correspondence: ghcdz@163.com

'State Key Laboratory of Plateau Ecology and Agriculture, Qinghai University, Xining 810016, China

${ }^{2}$ Academy of Agricultural and Forestry Sciences, Qinghai University, Xining 810016, China

Full list of author information is available at the end of the article
}

colors, including yellow, blue, purple, and black [3]. The use of colored grains in cereal-based functional foods has been considered based on their high levels of natural antioxidants such as phenolic compounds, anthocyanins and essential amino acids [4]. Colored barley varieties have been found to be more suitable for health than the standard (yellow) variety because these produce pearling fractions that are rich in bioactive compounds [5]. The development of barley flour, which itself has antioxidant properties, is used in various mainstream foods such as breads, muffins, noodles, and pasta [6]. The breeding of colored barley varieties has become an increasingly pertinent issue in agriculture in recent years.

Seed coat color is thought to be associated with the synthesis of anthocyanins, which are flavonoids present 
in plants [7]. The yellow color of barley is attributed to proanthocyanidins synthesized in the seed coat (testa layer) [8]. The purple color is associated with anthocyanins synthesized in the pericarp and glumes [9]. In barley, a number of mutants that lack anthocyanins or proanthocyanidins (designated ant mutants) have been documented. The Ant loci, known as anthocyanin or proanthocyanidin synthesis genes, are classified as Ant1 to Ant30 [10]. Flavonoid biosynthesis and the associated metabolic pathways have been studied in barley [11]. Also some candidate genes have been identified. Ant1 $(H v C 1)$, which is located on chromosome $7 \mathrm{H}$, reduces stem anthocyanin content [12]. Ant2 (HvbHLH1), which is located on chromosome $2 \mathrm{HL}$, regulates anthocyanin pigmentation in the auricles, awns, and lemmata. It is not involved, however, in grain proanthocyanidin pigmentation in grains [13]. Ant17 (flavanone 3-hydroxylase; F3H), which is located on chromosome $2 \mathrm{HL}$, has been observed in pigmented tissues. It is not found in nonpigmented roots and stems [14]. Ant18 (dihydroflavonol 4-reductase; DFR), which is located on chromosome $3 \mathrm{HL}$, is involved in both proanthocyanidin and anthocyanin synthesis [14, 15]. Ant28 (Hvmyb10), which is located on chromosome 3HL, specifically regulates proanthocyanidin synthesis for grain color and dormancy [10]. Although these genes that are involved in the biosynthesis of anthocyanins in barley have been identified, their association with seed color in hulless barley remains unclear.

Major obstacles to traditional methods for marker development include low efficiency and high cost of generating high-density genetic linkage maps [16]. High-throughput sequencing technology has enabled the determination of hulless barley genome sequences [17]. Therefore, accelerating the process and efficiency of molecular marker-assisted breeding in this plant species is important. A high-density genetic map is a valuable tool in genomic and genetic applications and especially in fine mapping [18, 19]. Genotyping-by-sequencing (GBS), a simple and relatively inexpensive procedure, has reduced the complexity of mapping because it is particularly suitable for a large number of samples in genetic map construction [20-22]. Recent advances in GBS technology have allowed the identification of numerous genetic molecular markers at a reasonable cost. This has promoted the development of several high-throughput single-nucleotide polymorphism (SNP) genotyping methods [23].

The present study generated a large double haploid (DH) population from a cross between the Nierumuzha and Kunlun10. The seed coat of Kunlun10 is yellow and that of Nierumuzha is deep purple. Dense marker data was obtained for $298 \mathrm{DH}$ individuals using the GBS technology. We identified 3662 efficient SNP markers, which were ultimately refined into 1129 bin markers after screening. The seed coat color of individual plants was determined. This information was used to map the Psc of hulless barley. The candidate genes and significant loci detected in this study suggested that our approach is cost-effective for fine mapping and can identify rapidly other key phenotypic genes in hulless barley.

\section{Materials and methods \\ Plant materials and phenotyping}

A DH mapping population that consisted of 298 individuals was drawn from hulless barley Kunlun10 (yellow seed coat color) as males and Nierumuzha individuals (deep purple seed coat color) were drawn as females (Fig. 1a). Seed color was assessed using the Wanseen seed test system SC-G (Wseen, Hangzhou, China) combined with visual inspection. Mean trait values were averaged from up to three repeats from each genotype in the $\mathrm{DH}$ population. Linkage analysis of the seed color was derived from average color estimates.

\section{DNA extraction}

Young leaves were collected from seven-week-old plants of parents and progeny after germination in April 2016. The leaves were immediately placed in liquid nitrogen immediately and then stored at $-80{ }^{\circ} \mathrm{C}$. A plant genomic DNA kit (TIANGEN, Beijing, China) was used for genomic DNA isolation in accordance with the manufacturer's protocol. DNA concentration and purity were assessed using a Nano Photometer ${ }^{\circ}$ spectrophotometer (Implen, CA, US). DNA contamination and degradation were assessed on $1.0 \%$ agarose gels.

\section{Library construction}

A genotyping-by-sequencing (GBS) (Novogene, Beijing, China) was used in this study to develop the SNP markers. A GBS pre-design experiment was performed. The GBS library was constructed using a double digest of genomic DNA with a combination of HaeIII, MseI, and EcoRI enzymes. This was subsequent to adapters with barcodes, after which each sample was amplified in multiplex with selected fragments for library construction. A tight length range was selected (about $50 \mathrm{bp}$ ) to maintain a uniform sequence depth among different fragments.

\section{Illumina sequencing}

First, standard analysis of the raw data was conducted. The Illumina HiSeq ${ }^{\text {Th }}$ sequencing platform (Illumina, San Diego, CA, USA) was employed for double-stranded (paired-end) 150-bp sequencing. Next, we conducted advanced analyses based on the assessment of the original data and DNA library assembly followed by HiSeq sequencing with removal of reads with low-quality base calls or uncalled bases. Second, we determined the number of reads digested by $M s e \mathrm{I}$ at both ends of each 


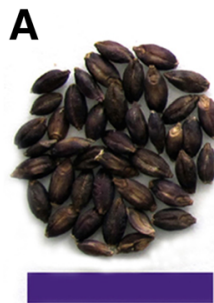

deep purple

Nierumuzha

B

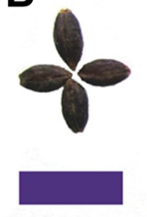

deep purple phenotype of deep purple, purple, light purple, and yellow color seeds
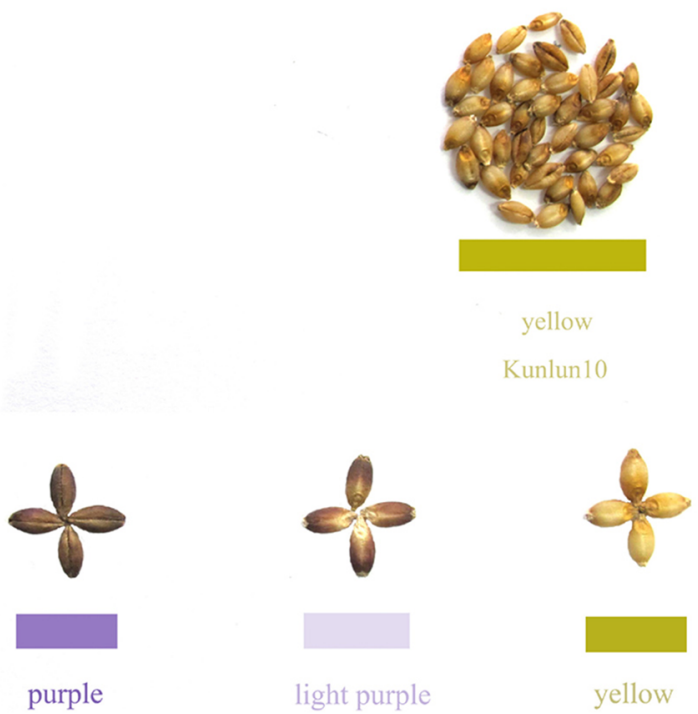

Fig. 1 The phenotype pictures of female, male and four types of seed coat color. a The seed coat color of Nierumuzha and Kunlun10; b The

screened read in the progeny subjected to GBS-Seq analysis. We then discarded reads that did not contain these restriction sites. The specific reads were recorded as well as the ratio of the total number of reads to the number of enzyme captured reads. Finally, using an Illumina high-throughput sequencing platform, pair-end sequencing was performed on the selected tags, followed by SNP genotyping and evaluation followed.

\section{Reference genome mapping}

For GBS, each sample was sequenced and then compared to the reference genome. Paired-end (PE) reads of the clean data from both the parent and offspring were compared to the reference genome by Burrows-Wheeler Aligner (BWA) software. SAM tools software [24] was used to create SAM/ BAM format files, which were used to detect mutations, and coverage was determined using Perl scripts. A linkage map was constructed that was based on the hulless barley genome database (reference genome, ftp://ftp.ensemblgenomes.org/ pub/release-29/plants/fasta/hordeum_vulgare/dna/).

\section{Sequence data analysis and SNP identification}

The barcodes were used to sort the sequences of each sample. To ensure that the reads were without artificial bias and were reliable (low-quality paired reads, which are mainly caused by base-calling duplicates and adapter contamination), raw data (raw reads) in FASTQ format were first processed in the subsequent analyses in a series of quality control (QC) procedures using in-house $C$ scripts. The QC standards were as follows: (1) removal of reads with $>50 \%$ bases having Phred quality $<5$; (2) removal of reads with $\geq 10 \%$ unidentified nucleotides $(\mathrm{N})$; (3) removal of reads containing the enzyme HaeIII and EcoRI enzyme restriction sites; and (4) Removal of reads with $>10 \mathrm{nt}$ aligned to the adapter, allowing $\leq 10 \%$ mismatches.

SNP calling in parents and progeny was performed using the SAMtools software [25]. The SNPs and types of transversions or transitions were counted. A Perl script was then employed to filter the SNPs that had more than two genotypes. The parental polymorphic markers were arranged into eight segregation patterns $(h k \times h k$, aa $\times b b$, $\mathrm{nn} \times \mathrm{np}, \mathrm{cc} \times \mathrm{ab}, \mathrm{ab} \times \mathrm{cc}, \mathrm{lm} \times \mathrm{ll}$, ab $\times \mathrm{cd}$ and ef $\times \mathrm{eg}$ ) according to the cross pollination (CP) model employed by JoinMap 4.0 software [26]. Segregation patterns were selected for the genetic mapping of offspring.

\section{Linkage map construction and linkage analysis}

Prior to map construction, the markers with integrity $>75 \%$, segregation distortion $(P<0.001)$, or that were in possession of abnormal bases were filtered. The segregation pattern aa $x$ bb was used for map construction using JoinMap 4.0. To calculate the marker distances, a regression algorithm, three times circulation sequence, and Kosambi [27] mapping function were used. LOD values were within the range of 2.0-10.0. The integrated map was computed for the male and female parents using the combined group for map integration function in the MergeMap software. A Perl script SVG was used to visualize the exported maps. Heat maps were constructed to evaluate those maps. Linkage analysis was conducted using an LOD threshold of 1000 permutations and a $P \leq 0.05$. A LOD score of 3.2 was set as the minimum for 1000 permutations to declare that any particular genomic region contained a locus. All candidate genes were categorized according to gene annotations by Swiss-Prot, 
TrEMBL, the Kyoto Encyclopedia of Genes and Genomes (KEGG) analysis.

\section{Results}

Resequencing of parental lines and GBS of the DH lines

The Nierumuzha and Kunlun10 parents were sequenced at effective sequencing depths of about 24.55-fold and 24.19-fold, respectively. 13,682,278 reads of Nierumuzha and 13,395,851 reads of Kunlun10 were mapped to the barley genome. The mapping rates were $96.81 \%$ and $96.72 \%$, respectively (Table 1 ). Finally, 224,008 polymorphic loci were identified. The marker genotype data are summarized in Table 2 . Only the genotype aa $\times \mathrm{bb}$, consisting of 20,615 markers between Nierumuzha and Kunlun10, was used in the subsequent for further analysis (Fig. 2).

The DH lines were then genotyped using the GBS technology. The average number of raw individual reads of the 298 hulless barley lines was about $1.6 \mathrm{~Gb}$. This yielded $490.07 \mathrm{~Gb}$ of high-quality sequence reads (Q20 $\geq 95 \%, Q 30 \% \geq 88 \%)$ and a normal GC content among the reads. The average MseI enzyme capture rate was $93.14 \%$ across the GBS of the $298 \mathrm{DH}$ lines, indicating that enzyme digestion was high quality. The average coverage of the 298 offspring was $96.93 \%$ of the whole genome, with 21.11 read depth at $8.38 \%$ (coverage at least $1 \times$ ) and $3.76 \%$ (coverage at least $4 \times$ ) of sites. The screened genotypes contained all of the markers in more than $75 \%$ of individual lines. Therefore, at least 227 of the 298 progeny lines contained all the markers.

\section{Genotyping of the progeny and selection of genetic markers}

The low-coverage sequences of the DH lines (coverage under 75\%) were filtered out. This left 7028 markers out of the original 20,615. Markers with significant distortion $(P<0.001)$ were filtered, and a total of 1549 markers were retained in total for identification of bin markers.

\section{Genetic linkage map with bin markers}

Unlinked markers were filtered out, and 3662 SNPs (1129 bin markers) were mapped to seven linkage maps using Joinmap 4.0. A high-density genetic map was constructed after these 1129 bin markers were mapped onto the seven
Table 2 Marker types

\begin{tabular}{ll}
\hline Marker type $^{a}$ & Numbers $^{b}$ \\
\hline $\mathrm{hk} \times \mathrm{hk}$ & 141,005 \\
aaxbb & 20,615 \\
$\mathrm{nn} \times \mathrm{np}$ & 30,054 \\
$\mathrm{cc} \times \mathrm{ab}$ & 7 \\
$\mathrm{ab} \times \mathrm{cc}$ & 6 \\
$\mathrm{Im} \times \|$ & 30,310 \\
$\mathrm{ab} \times \mathrm{cd}$ & 1 \\
ef $\times$ eg & 2010 \\
Total markers: & 224,008 \\
\hline
\end{tabular}

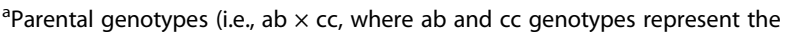
male and female parents)

${ }^{\mathrm{b}}$ Total number of markers of each type

chromosomes of hulless barley. The genetic maps were $645.56 \mathrm{cM}$ in length with an average distance of $0.57 \mathrm{cM}$ between the markers. $3 \mathrm{H}$ was the largest group among the seven linkage groups, consisting of 182 markers, and a genetic length of $127.21 \mathrm{cM}$. $1 \mathrm{H}$ was the smallest group, with 103 markers, and a genetic length of $60.21 \mathrm{cM}$ (Table 3, Fig. 3). A total of 1124 gaps were detected between markers. Among these, 11,102 gaps (98.04\%) were $<5 \mathrm{cM}$, 15 gaps were between 5 and $10 \mathrm{cM}$ in size, and only seven gaps were between 10 and $20 \mathrm{cM}$ in size. However, there were no gaps over $20 \mathrm{cM}$ in size were observed in any of the chromosomes (Table 4).

\section{Map quality validation}

A co-linearity analysis indicated that most of the markers in the linkage groups were consistent with the hulless barley reference genome, indicating that our estimation of genetic recombination rate was highly accurate (Fig. 4). The genetic map that was constructed with the SNP markers discovered in the GBS-Seq analysis had sufficient coverage throughout the barley genome. Most of the SNP loci on the linkage map were in the same order as those on the corresponding chromosomes of the physical map of the hulless barley genome.

Phenotypic features of DH lines and linkage analysis of Psc A DH population of 298 offspring from Nierumuzha $x$ Kunlun10 was used in this study. The seed coat color of

Table 1 Sequence depth and coverage statistics

\begin{tabular}{lllllll}
\hline Sample & Clean reads $^{\mathrm{a}}$ & Mapping reads $^{\mathrm{b}}$ & Mapping rate & Average depth $^{\mathrm{c}}$ & ${\text { Coverage } 1 \mathrm{x}^{\mathrm{e}}}_{\text {Coverage4 }^{\mathrm{f}}}$ \\
\hline Kunlun10 & $13,850,762$ & $13,395,851$ & 96.72 & 24.19 & 8.33 & 4.33 \\
Nierumuzha & $14,133,242$ & $13,682,278$ & 96.81 & 24.55 & 8.39 & 4.37 \\
\hline
\end{tabular}

${ }^{a}$ Number of reads used for the alignment

${ }^{\mathrm{b}}$ Number of clean reads that mapped to the reference genome

'The percentage of reads that mapped to the genome

${ }^{\mathrm{d}}$ Average sequencing depth

ePercentage of the reference genome with at least 19 coverage

${ }^{f}$ Percentage of the reference genome with at least 49 coverage 


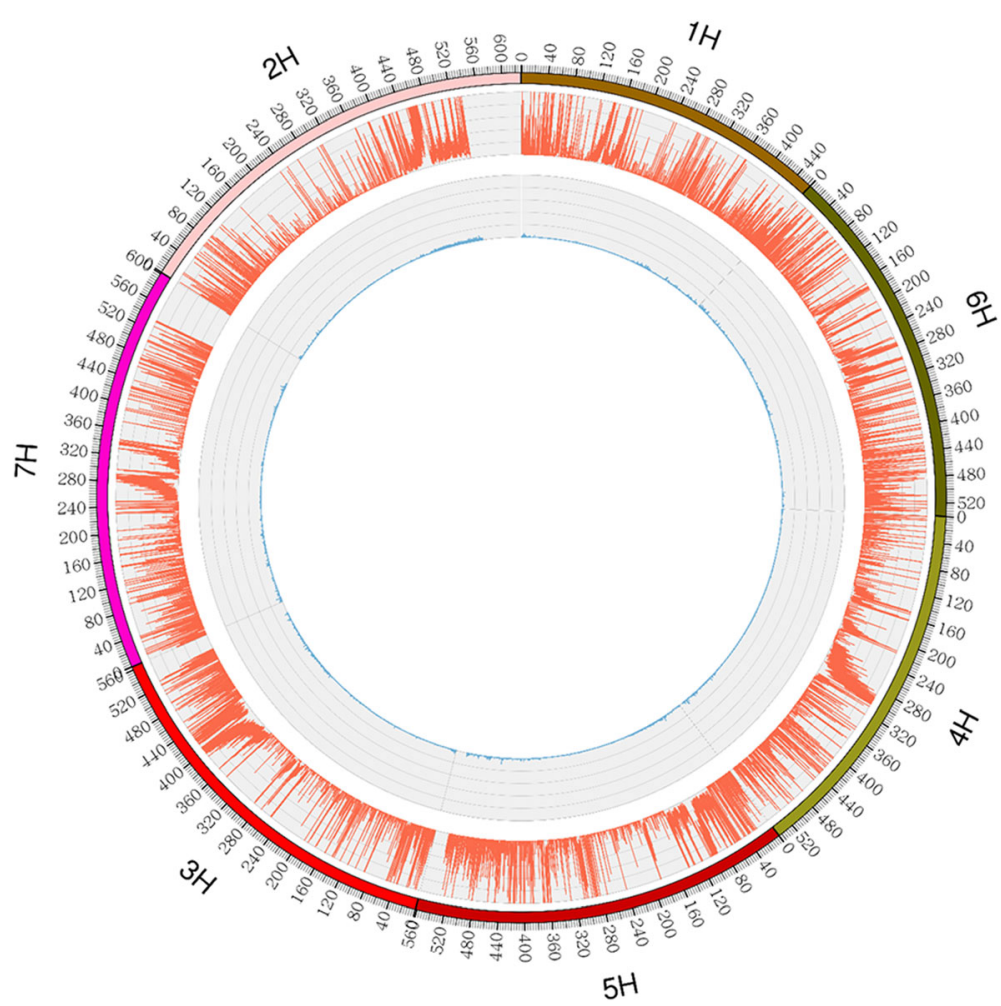

Fig. 2 Distribution of SNPs and the aa $\times$ bb genotype throughout the Nierumuzha and Kunlun10 genome-wide. The outermost box that includes a scale represents the seven chromosomes of hulless barley. The orange histogram represents the density of SNPs that are polymorphic between Kunlun10 and Nierumuzha. The green histogram represents the density of aa $\times$ bb genotype SNPs that are polymorphic between Kunlun 10 and Nierumuzha

offspring ranged from deep purple to yellow gradually (Additional file 1). We divided them artificially into four categories according to the result of Wanseen seed test system SC-G (27 deep purple seeds, 68 purple seeds, 133 light purple seeds, and 70 yellow seeds) (Fig. 1b) [28]. Linkage analysis was based on the phenotypic data for Psc in DH lines at a LOD of 3.2. A total of 5 loci for Psc were identified: one locus on $4 \mathrm{H}$, explaining $3.79 \%$ and four loci on $7 \mathrm{H}$ (Fig. 5), explaining 13.64\%, 17.56\%, 23.86\%, and $15.26 \%$ of phenotypic variation, respectively. The locus peak positions were located on $10.91 \mathrm{cM}(4 \mathrm{H}), 71.81 \mathrm{cM}$ (7H), $77.41 \mathrm{cM}(7 \mathrm{H}), 84.61 \mathrm{cM}(7 \mathrm{H})$, and $90.61 \mathrm{cM}(7 \mathrm{H})$,

Table 3 Genetic linkage group statistics

\begin{tabular}{|c|c|c|c|c|c|c|}
\hline$\overline{\mathrm{Chr}^{\mathrm{a}}}$ & $\mathrm{PD}(\mathrm{bp})^{\mathrm{b}}$ & Bin markers ${ }^{c}$ & SNP $^{d}$ & $\mathrm{GD}(\mathrm{cM})^{\mathrm{e}}$ & Average distance $(\mathrm{cM})^{f}$ & Max. gap $(\mathrm{cM})^{9}$ \\
\hline$\overline{1 H}$ & $464,124,043$ & 103 & 230 & 60.21 & 0.58 & 4.67 \\
\hline $2 \mathrm{H}$ & $628,342,783$ & 301 & 1340 & 81.43 & 0.27 & 10.82 \\
\hline $3 \mathrm{H}$ & $564,427,874$ & 182 & 406 & 127.21 & 0.70 & 16.02 \\
\hline $4 \mathrm{H}$ & $544,168,226$ & 117 & 312 & 81.36 & 0.70 & 11.05 \\
\hline $5 \mathrm{H}$ & $561,411,686$ & 127 & 240 & 101.94 & 0.80 & 12.86 \\
\hline $6 \mathrm{H}$ & $538,755,036$ & 111 & 541 & 98.76 & 0.89 & 9.36 \\
\hline $7 \mathrm{H}$ & $601,597,413$ & 188 & 593 & 94.63 & 0.50 & 14.84 \\
\hline Total & $3,902,827,061$ & 1129 & 3662 & 645.56 & 0.57 & 16.02 \\
\hline
\end{tabular}

${ }^{\mathrm{a} C h r o m o s o m e ~ n u m b e r ~}$

${ }^{\mathrm{b}}$ Total physical length of the chromosomes (bp)

cNumber of bin markers

${ }^{\mathrm{d}}$ Single Nucleotide Polymorphism

'Total genetic distance of chromosomes (cM)

${ }^{f}$ Average genetic distance between markers (cM)

${ }^{\mathrm{g}}$ Maximum gap between markers (cM) 


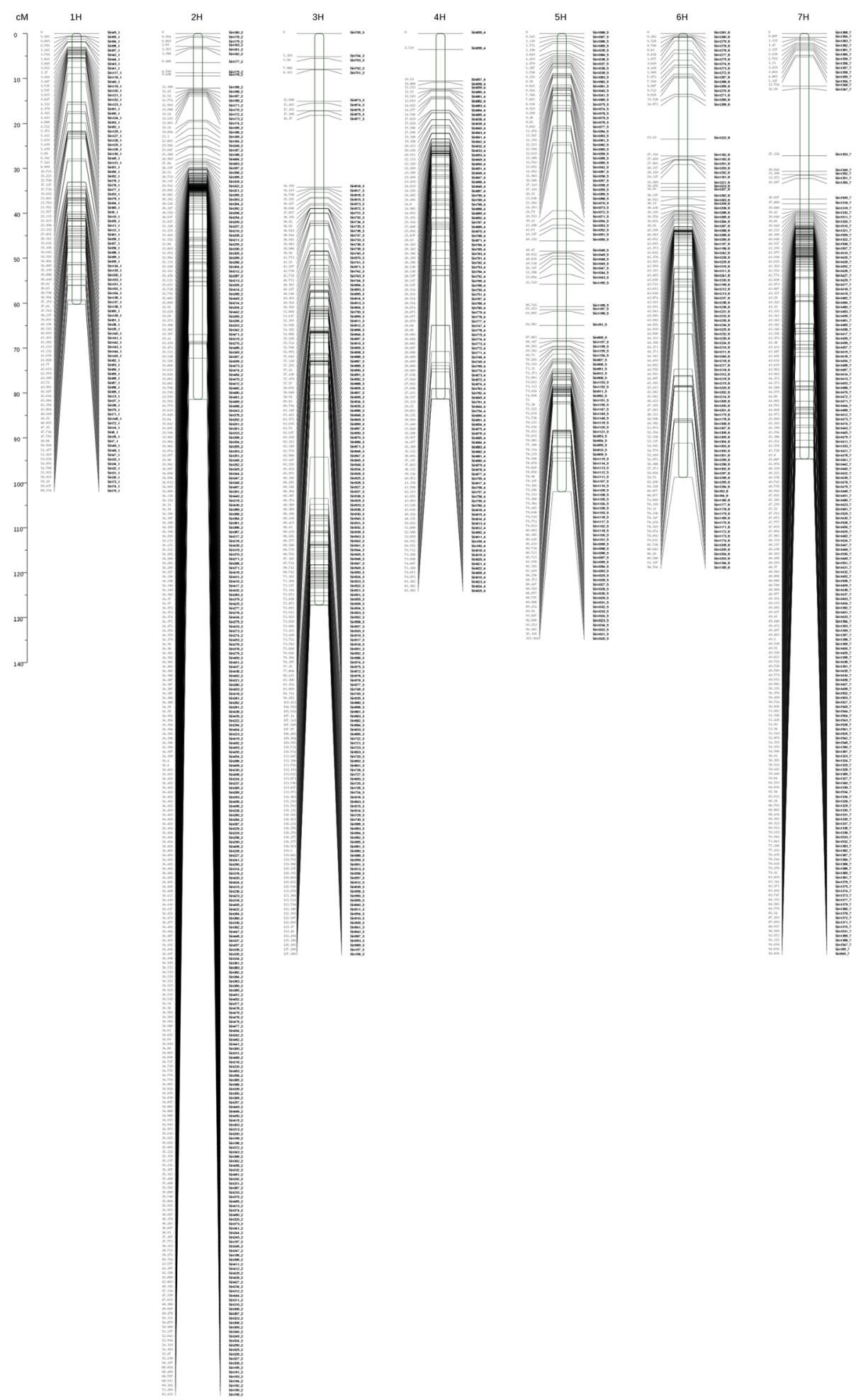

Fig. 3 Distribution map of the linkage groups. Note, the $x$-axis is the chromosome number, the $y$-axis is the genetic distance (in cM), and the black line is the bin marker 
Table 4 Linkage map gap statistics

\begin{tabular}{llllll}
\hline $\mathrm{Chr}^{\mathrm{a}}$ & $<5 \mathrm{cM}$ & 5 to10 cM & 10 to20 cM & $>20 \mathrm{cM}$ & ratio \\
\hline $1 \mathrm{H}$ & 103 & 0 & 0 & 0 & 100.00 \\
$2 \mathrm{H}$ & 297 & 2 & 1 & 0 & 99.00 \\
$3 \mathrm{H}$ & 176 & 3 & 2 & 0 & 97.24 \\
$4 \mathrm{H}$ & 113 & 2 & 1 & 0 & 97.41 \\
$5 \mathrm{H}$ & 123 & 2 & 1 & 0 & 97.62 \\
$6 \mathrm{H}$ & 106 & 4 & 0 & 0 & 96.36 \\
$7 \mathrm{H}$ & 184 & 2 & 1 & 0 & 98.40 \\
Total & 1102 & 15 & 7 & 0 & 98.00 \\
\hline
\end{tabular}

${ }^{\mathrm{a} C h r o m o s o m e ~ n u m b e r ~}$

respectively, and were located within the marker intervals of $4 \mathrm{H}$-bin 857 to $4 \mathrm{H}$ - bin863 (275 genes), $7 \mathrm{H}$ - bin 1533 to 7H- bin1532 (57 genes), 7H- bin1383 to 7H- bin1384 (60 genes), $7 \mathrm{H}$ - bin1377 to $7 \mathrm{H}$ - bin1372 (248 genes), and $7 \mathrm{H}-$ bin1370 to $7 \mathrm{H}$ - bin1369 (189 genes) (Table 5).

\section{Prediction of candidate genes}

Five structural candidate genes and one regulatory factor related to flavonoid or anthocyanin biosynthesis were identified in the Psc regions within the loci (Additional file 2) according to the barley gene annotation database accessible at TrEMBL, NCBI, KEGG and Swiss-Prot. The candidate gene MLOC_6177, located on chromosome 7H (71.00$73.10 \mathrm{cM}$, see Additional file 3), was annotated to an uncharacterized protein in barley in TrEMBL and flavonoid 3'-monooxygenase $\left(F 3^{\prime} M\right)$ in Aegilops (GenBank Accession Number XM_020332250.1) in KEGG and Swiss-Prot. MLOC_71630 located on chromosome $7 \mathrm{H}$ (77.30-78.50 cM, see Additional file 4), was annotated to an uncharacterized protein in barley at TrEMBL and 2-hydroxyisoflavanone dehydratase $(H I D)$ in Aegilops in Swissprot. MLOC_62096 located on chromosome $7 \mathrm{H}$ (84.50-86.00 cM, see Additional file 5), was annotated to anthocyanidin 3-O-glucoside 2' '-O-xylosyltransferase (UF3GT) in barley in Swiss-Prot. MLOC_ 38343 located on chromosome $7 \mathrm{H}(84.50-86.00 \mathrm{cM}$, see Additional file 5), was annotated anthocyanidin/flavonoid 3-O-glucosyltransferase (UFGT) in barley in Swiss-Prot. MLOC_32012 located on chromosome 7H (90.50$90.70 \mathrm{cM}$, see Additional file 6), was annotated to an uncharacterized protein in barley in TrEMBL and malonyl -coenzyme: anthocyanin 5-O-glucoside-6" '-O-malonyltransferase (5MAT) in Aegilops in Swiss-Prot. MLOC_ 6171 located on chromosome $7 \mathrm{H}(84.50-86.00 \mathrm{cM}$, see Additional file 5), was annotated to an uncharacterized protein in barley in TrEMBL, but anthocyanin regulatory $\mathrm{C} 1$ protein $(A n t 1)$ in barley in NCBI.

\section{Discussion}

Linkage analysis is an efficient way to analyze some important traits in barley molecular breeding $[29,30]$. The

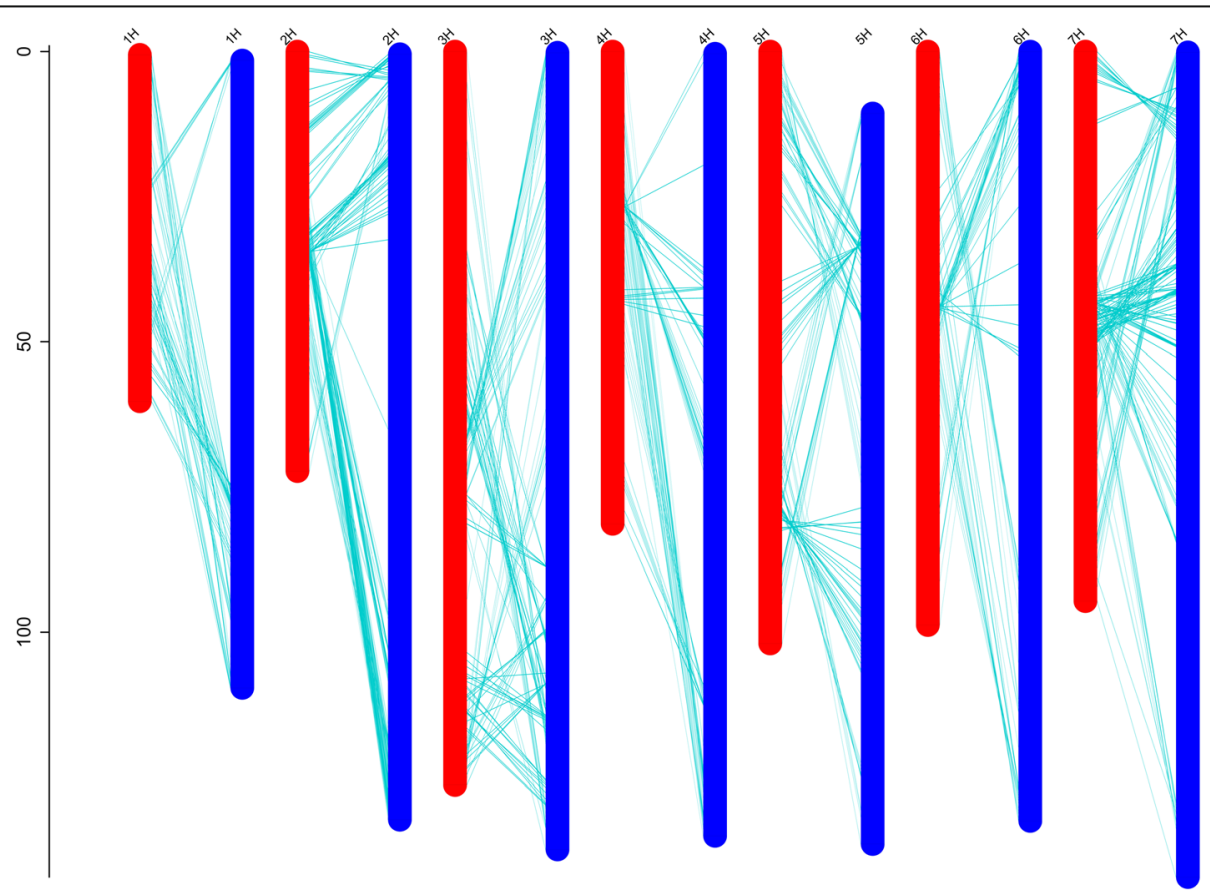

Fig. 4 The physical map and genetic linkage map. Note, the $x$-axis is the chromosome number and the $y$-axis is the genetic distance (in $c M$ ). The genetic map is shown in red, the physical map is in blue, and the green line shows the position of each marker on the genetic map and the physical map 


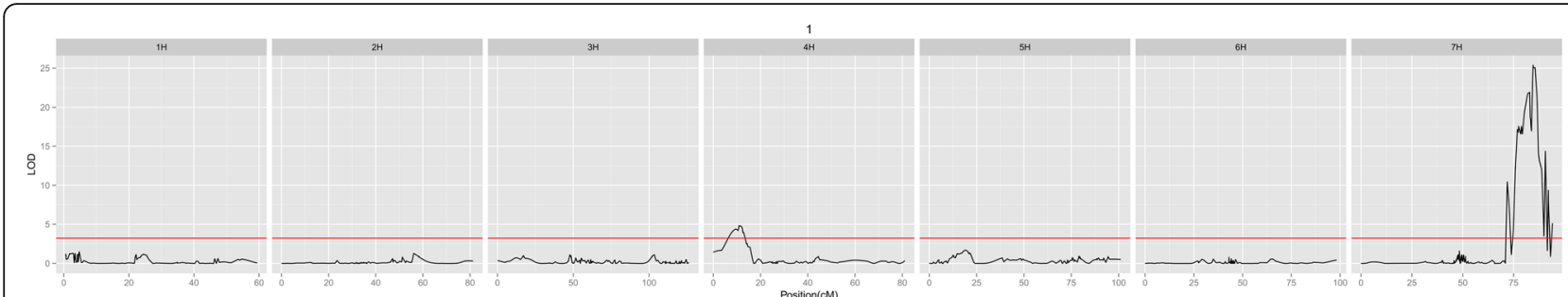

Fig. 5 Linkage analysis of PSC in hulless barley among all the linkage groups. Note: the x-axis is the genetic distance of each linkage group, the $y$-axis is the physical position of bin markers against LOD scores and the red line is the threshold LOD value as determined by a permutation test: $1 \sim 7$, seven linkage groups

quality of genetic maps, however, can significantly affect the accuracy of gene mapping. This increases the density of markers distributed around the entire genome, which can improve the resolution of genetic maps [31-33]. SSR, one of the traditional gene mapping methods, was used in hulless barley, but the number of SSR markers is limited. Meanwhile, no markers were sufficiently close to key traits to ensure reliable detection after exhaustively testing [34]. GBS is a fast, efficient, informative, and cost-effective strategy for SNP discovery, genetic linkage map construction, and genotyping [35]. In this study we used a combination of three restriction enzymes (HaeIII, MseI, and EcoRI) for GBS library construction. MseI recognizes a 4-bp restriction site (TTAA). It also has a higher distribution frequency in the Chinese jujube (Ziziphusjujuba) genome. The use of two additional enzymes, namely, EcoRI and HaeIII, was performed to further digest the fragments after MseI. The three restriction enzyme combination improved the efficiency of GBS by increasing sequencing depth, number of tags, and genome coverage. This combination also allowed the detection of suitable regions for targeted fragments [36]. The present study generated the first high-density genetic map of hulless barely using the GBS technology. A total of $490.07 \mathrm{~Gb}$ raw sequencing data and $96.93 \%$ of clean data were mapped to unique positions on the reference genome. We were able to cost-effectively genotype 3662 SNPs with 1129 bin markers with an average distance of $0.57 \mathrm{cM}$ in
300 samples (2 parents and 298 offspring) of hulless barely using the GBS technology. Such a high-density linkage map will likely be a valuable resource for genomic analyses and fine-scale gene mapping in hulless barely.

The inheritance of the purple pericarp and lemma has been studied with inconsistent results up to date. Woodward and Thieret [37] obtained 3:1 ratios of purple and nonpurple seeds from 28 crosses of "purple" $\times$ "nonpurple" barley genotypes, and 9:7 ratios from the crosses in which both parents were non-purple seeds, indicating a two-factor inheritance. To simplify the nomenclature, previously reported symbols were dropped and Pre1 and Pre 2 symbols were used for $\mathrm{C}$, c, and P, p, located on $1 \mathrm{H}$ and $2 \mathrm{H}$, respectively [38]. In this study we found 298 offspring from Nierumuzha $\times$ Kunlun10 seed coat colors range from deep purple to yellow gradually. In order to shorten the distinguish time of breeding in future, we divided them artificially into four categories: deep purple, purple, light purple and yellow. We thus mapped Psc as a qualitative trait using the GBS linkage map. The major Psc loci were located on chromosome $4 \mathrm{H}$ (1 locus) and $7 \mathrm{H}$ (4 loci). Previous studies have indicated that seed coat color of barley was located on chromosomes $2 \mathrm{H} \mathrm{[38]} \mathrm{and} 4 \mathrm{H}$ [13] at a genetic distance greater than $10 \mathrm{cM}$. In this study, five loci were detected on chromosomes $4 \mathrm{H}$ and $7 \mathrm{H}$ that one explained $3.79 \%$ (length $3.3 \mathrm{cM}$ ) of the phenotypic variation, whereas the other four $(71.00 \mathrm{cM}-90.70 \mathrm{cM})$ accounted for $13.64 \%$ (length $2.1 \mathrm{cM}$ ), 17.56\% (length

Table 5 Linkage analysis of PSC

\begin{tabular}{|c|c|c|c|c|c|c|c|c|c|}
\hline Site name & $\mathrm{Chr}^{\mathrm{a}}$ & LOD peak & $\begin{array}{l}\text { Position } \\
\text { (cM) }\end{array}$ & $99 \% \mathrm{Cl}(\mathrm{cM})^{\mathrm{b}}$ & $R^{2}(\%)^{c}$ & $\begin{array}{l}\text { Additive } \\
\text { effect }\end{array}$ & $\begin{array}{l}\text { Left } \\
\text { marker }^{\mathrm{d}}\end{array}$ & $\begin{array}{l}\text { Right } \\
\text { marker }\end{array}$ & $\begin{array}{l}\text { Gene } \\
\text { number }\end{array}$ \\
\hline$\overline{P S C-1}$ & $4 \mathrm{H}$ & 4.81 & 10.91 & $10.5-13.8$ & 3.79 & 0.2779 & bin857 & bin863 & 275 \\
\hline PsC-2 & $7 \mathrm{H}$ & 10.45 & 71.81 & $71-73.1$ & 13.64 & -0.376 & bin1533 & bin1532 & 57 \\
\hline PSC-3 & $7 \mathrm{H}$ & 17.57 & 77.41 & $77.3-78.5$ & 17.56 & 0.443 & bin1383 & bin1384 & 60 \\
\hline Psc-4 & $7 \mathrm{H}$ & 25.37 & 84.61 & $84.5-86$ & 23.86 & 0.5598 & bin1377 & $\operatorname{bin} 1372$ & 248 \\
\hline PSC-5 & $7 \mathrm{H}$ & 14.34 & 90.61 & $90.5-90.7$ & 15.26 & -0.3707 & bin1370 & bin1369 & 189 \\
\hline
\end{tabular}

${ }^{\mathrm{a}}$ Chromosome number

${ }^{\mathrm{b}} 99 \%$ confidence interval for site length

'Proportion of phenotypic variation explained by each site

'Left boundary markers for each site

${ }^{\mathrm{e}}$ Right boundary markers for each site 
$1.2 \mathrm{cM}$ ), $23.86 \%$ (length $1.5 \mathrm{cM}$ ), and15.26\% (length $0.2 \mathrm{cM})$ of the phenotypic variation according to the high-resolution map (Table 5). There are 23 bin markers between $70.944 \mathrm{cM}$ and $92.072 \mathrm{cM}$ (bin1533-bin1369) on $7 \mathrm{H}$, and the average marker density is $0.92 \mathrm{cM}$. The average gap is $0.746 \mathrm{cM}$ except the largest one $(5.455 \mathrm{cM})$ between qPSC-2 and qPSC-3 (bin1532-bin1383) (Additional file 7). In order to avoid the wrong judgment in this region, we re-tested candidate genes according to gene annotations by Swissprot, TrEMBL, and the Kyoto Encyclopedia of Genes and Genomes (KEGG) analysis, but didn't found new ones.

Seed coat color is thought to be associated with the synthesis of anthocyanins. Anthocyanin biosynthesis is well characterized at the enzymatic, genetic, and production levels. These structural genes can be separated into two categories: those of the late flavonoid biosynthetic pathway, including anthyocyanidin synthase (ANS), dihydroflavonol 4-reductase (DFR), UDP-sugar: flavonoid-3-O-glucosyltransferase (UFGT), and flavonoid-5-O-glucosyltransferase (UF5GT) [39], and those of the early flavonoid biosynthetic pathway, including chalcone synthase $(\mathrm{CHS})$, chalcone isomerase $(\mathrm{CHI})$, phenylalanine ammonia-lyase $(P A L)$, flavonoid 3-hydroxylase $(F 3 H)$, and flavonoid 3'-hydroxylase $\left(F 3^{\prime} H\right)$. Shoeva et al. identified the Ant2 gene and showed that the mRNA levels of flavonoid biosynthesis structural genes $C H S, C H I, F 3 H, D F R, F 3^{\prime} H$, $A N S$, and the regulatory gene $A N T 2$ were higher in purple barley than in yellow barley, as indicated by qRT-PCR [3]. Even though the genes involved in anthocyanin biosynthesis have been identified, their relation to Psc in hulless barley is currently unknown. In this study, some structural candidate genes and regulatory factors related to flavonoid or anthocyanin biosynthesis were identified in the Psc regions by gene annotation. Sequence analysis showed the nucleotide sequence of MLOC_6177 from hulless barley to be $99 \%$ homologous with a predicted protein gene NIASHv2048J10 (GenBank Accession Number AK366933.1) of barley and 95\% homologous with $F 3^{\prime} M$ of Arabidopsis (GenBank Accession Number AK366933.1), respectively. $F 3^{\prime} M$ belongs to the cytochrome $\mathrm{P} 450$ family and is related to flavonoid biosynthesis [40]. The nucleotide sequence of MLOC_71630 from hulless barley was $99 \%$ homologous with a predicted protein gene NIASHv1124H04 of barley (GenBank Accession Number AK360742.1) and 92\% homologous with 2-hydroxyisoflavanone dehydratase gene of Aegilops (GenBank Accession Number XM_020315035.1). The co-action of a HID and 2-hydroxyisoflavanone synthase (IFS) produces flavone from flavanone, which is related to isoflavone biosynthesis [41]. The nucleotide sequence of MLOC_62096 from hulless barley was 99\% homologous with UF3GT of barley (GenBank Accession Number AK358154.1). UF3GT contributes to the last few steps in anthocyanin biosynthesis by converting cyanidin 3-O-xylosyl (1->2) glucoside. It can use 3-O-glucosylated anthocyanidins and uridine diphosphate (UDP)-xylose as substrates [42]. The nucleotide sequence of MLOC_38343 from hulless barley was 99\% homologous with UFGT of barley (GenBank Accession Number X15694.1). UFGT is involved in the anthocyanin biosynthesis pathway, which forms part of pigment biosynthesis [42]. The nucleotide sequence of MLOC_32012 from hulless barley was $92 \%$ homologous with $5 M A T$ of Aegilops (GenBank Accession Number XM_020296576.1). $5 M A T$ is involved in later reactions in anthocyanin modification [43]. The nucleotide sequence of MLOC_6171 from hulless barley was $99 \%$ homologous with Ant1 of barley (GenBank Accession Number KP265979.1). Ant1 can act as a trans-acting factor (MYB related family protein) that controls the expression of genes involved in anthocyanin biosynthesis and regulates the expression of at least three structural genes: dihydroflavonol reductase, chalcone synthase, and flavonol $\mathrm{O}_{3}$ glucosyltransferase [44]. Regulatory factors such as MYB, bHLH and WD40 proteins [45] primarily control anthocyanin biosynthesis, as well as a series of structural genes (enzymes). In addition to Ant1, we also discovered some other transcription factors including bHLH69, bHLH82, bHLH96, MYB39, WDR, TRAB1 and IND, etc. The relationship between transcription factors and anthocyanin biosynthesis still requires further validation. Also, the function of the above-mentioned annotation genes need further study.

\section{Conclusion}

A high-density genetic linkage map was constructed using the GBS method. The linkage map contained seven linkage groups with a low inter-marker distance. This high-density linkage map can serve as a foundation for obtaining additional genetic knowledge of hulless barley. Five loci for $P s c$ were identified and will be useful in marker-assisted selection studies for this important agronomic trait. Using linkage analysis and gene annotation, five structural candidate genes and one regulatory factor related to flavonoid and anthocyanin biosynthesis were identified. These genomic resources may also play an important role in genetic breeding studies and future whole-genome sequencing projects in hulless barley.

\section{Additional files}

Additional file 1: The phenotype pictures of female, male and four types of seed coat color. (JPG $267 \mathrm{~kb}$ )

Additional file 2: All candidate genes sequences. (DOCX $18 \mathrm{~kb}$ )

Additional file 3: Gene annotations of $7 \mathrm{H}$ within the region encompassing 71.00-73.10 cM. (XLSX 15 kb)

Additional file 4: Gene annotations of $7 \mathrm{H}$ within the region encompassing $77.30-78.50 \mathrm{cM}$. (XLSX $15 \mathrm{~kb})$ 
Additional file 5: Gene annotations of $7 \mathrm{H}$ within the region encompassing of 84.50-86.00 cM. (XLSX $30 \mathrm{~kb})$

Additional file 6: Gene annotations of $7 \mathrm{H}$ within the region encompassing 90.50-90.70 cM. (XLSX 25 kb)

Additional file 7: The marker information between bin1533 to bin1531 of 7H. (DOCX 16 kb)

\section{Abbreviations}

5MAT: Malonyl-coenzyme: -O-glucoside-6"'-O- malonyltransferase; ANS: Anthyocyanidin synthase; BWA: Burrows-wheeler aligner; $\mathrm{CHI}$ : Chalcone isomerase; CHS: Chalcone synthase; CP: Cross pollination;

DFR: Dihydroflavonol 4-reductase; $\mathrm{DH}$ : Doubled haploid lines; $F 3^{\prime} H$ : Flavonoid 3'-hydroxylase; F3'M: Flavonoid 3'-monooxygenase; F3H: Flavanone 3-hydroxylase; GBS: Genotyping by sequencing; IFS: 2-hydroxyisoflavanone synthase; LOD: Logarithm of odds; PAL: Phenylalanine ammonia-lyase; PE: Paired-end; PSC: Purple seed coat color; QC: Quality control; SNP: Single nucleotide polymorphism; UF5GT: Flavonoid-5-O-glucosyltransferase; UFGT: Anthocyanidin/ flavonoid 3-O-glucosyltransferase; UFGT: UDP-sugar: flavonoid-3-O-glucosyltransferase

\section{Acknowledgements}

We would like to thank Jianghua Huang and Ruiju Lu for DH of the Shanghai Academy of Agricultural Science, Novogene (https://en.novogene.com) for the practical conduction of the GBS analysis and Accdon (www.accdon.com) for editing this manuscript. We are grateful to the reviewers for their valuable comments.

\section{Funding}

This research was funded by the Natural Science Foundation of China (31660388), China Agriculture Research System (CARS-05), the Project of Qinghai Science and Technology Department (2016-ZJ-Y01and 2016-NK-131), and the Open Project of State Key Laboratory of Plateau Ecology and Agriculture, Qinghai University (2017-ZZ-14).

\section{Availability of data and materials}

The data supporting the findings of this study are available within the article.

\section{Authors' contributions}

$\mathrm{XHY}$ designed the research protocol, analyzed the data, carried out the experiment, and drafted the manuscript. YHY, JXY, and YXB assisted in the data analysis, supervised the protocol and prepared the manuscript. KLW and DZC finally approved the version to be published. All authors read and approved the final manuscript.

\section{Ethics approval and consent to participate}

Not applicable.

\section{Consent for publication}

Not applicable.

\section{Competing interests}

The authors declare that they have no competing interests.

\section{Publisher's note}

Springer Nature remains neutral with regard to jurisdictional claims in institutional affiliations and published maps.

\section{Author details}

${ }^{1}$ State Key Laboratory of Plateau Ecology and Agriculture, Qinghai University, Xining 810016, China. ${ }^{2}$ Academy of Agricultural and Forestry Sciences, Qinghai University, Xining 810016, China. ${ }^{3}$ Qinghai Key Laboratory of Hulless Barley Genetics and Breeding, Xining 810016, China. ${ }^{4}$ Qinghai Subcenter of National Hulless Barley Improvement, Xining 810016, China.
Received: 2 July 2018 Accepted: 18 October 2018

Published online: 17 November 2018

\section{References}

1. Liang JJ, Deng GD, Long H, Pan ZF, Wang CP, Cai P, Xu DL, Nimaand ZX, Yu MQ. Virus-induced silencing of genes encoding LEA protein in Tibetan hulless barley (Hordeumvulgare ssp. vulgare) and their relationship to drought tolerance. Mol Breeding. 2012;30(1):441-51.

2. Yan XY, Li JN, Fu FY, Jin MY, Chen L, Liu LZ. Co-location of seed oil content, seed hull content and seed coat color QTL in three different environments in Brassicanapus L. Euphytica. 2009;170(3):355-64.

3. Shoeva OY, Mock HP, Kukoeva Tatjana V, Borner A, Khlestkina EK. Regulation of the flavonoid biosynthesis pathway genesin purple and black grains of Hordeumvulgare. PLoS One. 2016;11(10):e0163782.

4. Abdel-Aal ES, Young JC, Rabalski I. Anthocyanin composition in black, blue, pink, purple and red cereal grains. J Agric Food Chem. 2006;54(13):4696-704

5. Bellido GG, Trust B. Anthocyanin composition and oxygen radical scavenging capacity (ORAC) of milled and pearled purple, black, and common barley. J Agric Food Chem. 2009;57(3):1022-8.

6. Madhujith T, Izydorczyk M, Shahidi F. Antioxidant properties of pearled barley fractions. J Agric Food Chem. 2006;54(9):3283-9.

7. Kim MJ, Jong NH, Aekim J, P ark JC, Kim MY, Kim JG, Lee SJ, Chun SC, Chung AM. Relationship between phenolic compounds, anthocyanins content and antioxidant activity in colored barley germplasm. J Agric Food Chem. 2007;55(12):4802-9.

8. Aastrup S, Outtrup H, Erdal K. Location of the proanthocyanidins in the barley grain. Carlsb Res Commun. 1984;49:105-9.

9. Harlan HV. Some distinctions in our cultivated barleys with reference to their use in plant breeding. U S Dept Agr Bull. 1914;137:38.

10. Himi E, Yamashita $Y$, Haruyama N, Yanagisawa T, Maekawa M, Taketa S. Ant28 gene for proanthocyanidin synthesis encoding the R2R3 MYB domain protein (Hvmyb10) highly affects grain dormancy in barley. Euphytica. 2012;188(1):141-51.

11. Badbro JS. Genetic control of flavonoid biosynthesis in barley. Hereditas 1993:119(2):187-204.

12. Himi $E$, Taketa S. Barley Ant17, encoding flavanone 3-hydroxylase $(F 3 H)$, is a promising target locus for attaining anthocyanin/ proanthocyanidin-free plants without pleiotropic reduction of grain dormancy. Genome. 2015;58(1):43-53.

13. Cockram J, White J, Zuluaga DL, Smith D, Comadran J, Macaulay M, Luo ZW, Kearsey MJ, Werner P, Marshall DF, et al. Genome-wide association mapping to candidate polymorphism resolution in the unsequenced barley genome. Proc Natl Acad Sci U S A. 2010;107(50):21611-6.

14. Peukert M, Weise S, Roder MS, Matthies IE. Development of SNP markers for genes of the phenylpropanoid pathway and their association to kernel and malting traits in barley. BMC Genet. 2013;14(1):97-112.

15. Kristiansen K, Rohde W. Structure of the Hordeumvulgare gene encoding dihydroflavonol-4-reductase and molecular analysis of ant18 mutants blocked in flavonoid synthesis. Mol Gen Genet. 1991;230(1-2):49-59.

16. Baxter SW, Davey JW, Johnston JS. Linkage mapping and comparative genomics using next-generation RAD sequencing of a non-model rganism. PLoS One. 2011;6(4):e19315.

17. Zeng $X Q$, Long $H$, Wang $Z$, Zhao SC, Tang $Y W$, Huang $Z Y$, Wang $Y L, X u Q J$, Mao LK, Deng GB, et al. The draft genome of Tibetan hulless barley revealsadaptive patterns to the high stressful Tibetan plateau. Proc Natl Acad Sci U S A. 2015;112(4):1095-100.

18. Guajardo V, Solis S, Sagredo B, Gainza F, Muñoz C, Gasic K, Hinrichsen P. Construction of high density sweet cherry (PrunusaviumL.) linkage maps using microsatellite markers and SNPs detected by genotyping-bysequencing(GBS). PLoS One. 2015;10(5):e0127750.

19. Jiang $B$, Liu W, Xie D, Peng Q, He X, Lin Y, Liang ZJ. High-density genetic map construction and gene mapping of pericarp color in wax gourd using specificlocus amplified fragment (SLAF) sequencing. BMC Genomics. 2015;16(1):1035.

20. Qi HK, Wang N, Qiao WQ, Xu QH, Zhou H, Shi JB, Yan GT, Huang Q. Construction of a high-density genetic map using genotyping by sequencing (GBS) for quantitative trait loci (QTL) analysis of three plant morphological traits in upland cotton (GossypiumhirsutumL.). Euphytica. 2017:213(4):83-99.

21. Wang JP, Li L, Zhang GF. A high-density SNP genetic linkage map and QTL analysis of growth-related traits in a hybrid family of oysters (Crassostreagigas $X$ Crassostreaangulata) using genotyping-by-sequencing. G3. 2016;6(5):1417-26. 
22. Zhou ZQ, Zhang CS, Zhou Y, Hao ZF, Wang ZH, Zeng X, et al. Genetic dissection of maize plantarchitecture with an ultra-high density binmap based on recombinant inbred lines. BMC Genomics. 2016;7:178-92.

23. Nie HT, Yan XW, Huo ZM, Jiang LW, Chen P, Liu H, Ding JF, Yang F, et al. Construction of a high-density genetic map and quantitative trait locus mapping in the manila clam Ruditapesphilippinarum. Sci Rep. 2017;7(1):229-37.

24. Li H, Durbin R. Fast and accurate short read alignment with burrowswheeler transform. Bioinformatics. 2009;25(14):1754-60.

25. Li H, Handsaker B, Wysoker A, Fennell T, Ruan J, Homer N, Marth G, Abecasis G, Durbin R. 1000 genome project data processing subgroup. The sequence alignment/map format and SAMtools. Bioinformatics. 2009;25(16):2078-9.

26. Van Ooijen JW. JoinMap ${ }^{\oplus}$ 4.0: software for the calculation of genetic linkage maps in experimental population. Wageningen. In: The Netherlands; 2006.

27. Kosambi DD. The estimation of map distance from recombination values. Ann Eugenics. 1943;12(1):172-5.

28. Yuki HC, Hiroki Y, Ogura R, Naomi S, Hitoshi S, Kiyoaki K. Genetic analysis and molecular mapping of genes controlling seed coat colour in adzuki bean (Vigna angularis). Euphytica. 2015;206(3):609-17.

29. Graner A, Bauer E, Kellermann A, Proeseler G, Wenzel G, Ordon F. RFLP analysis of resistance to barley yellow mosaic virus complex. Agronomie. 1995;15:475-9.

30. Roslinsky V, Eckstein PE, Raboy V, Rossnagel BG, Scoles GJ. Molecular marker development and linkage analysis in three low phytic acid barley (Hordeum vulgare) mutant lines. Mol Breeding. 2007;20(4):323-30.

31. Cynara CTR, Jasper PV, Anton V, Axel H, Martin M, Rients EN. Mapping resistance to powdery mildew in barley reveals a large-effect nonhost resistance QTL. Theor Appl Genet. 2018;131(5):1031-45.

32. Zhang XC, Shabala S, Koutoulis A, Shabala L, Zhou MX. Meta-analysis of major QTL for abiotic stress tolerance in barley and implications for barley breeding. Planta. 2017;245(2):283-95.

33. Fan CF, Zhai HJ, Wang HF, Yue YF, Zhang MH, Li JH, Wen SZ, Guo GG, Zeng YW, Ni ZF, You MS. Identification of QTLs controlling grain protein concentration using a high-density SNP and SSR linkage map in barley (Hordeum vulgare L.). BMC Plant Biol. 2017;17:122-45.

34. Eshghi R, Salayeva S, Ebrahimpour F, Rahimi M, Baraty M, Ojaghi J. Advanced-backcross QTL analysis in hulless barley: I. detection of exotic alleles for yield and yield components introgressed from Hordeum vulgare ssp. spontaneum. Intl Agri. Crop Sci. 2013;5(2):95-100

35. Liu H, Bayer M, Druka A, Russell JR, Hackett CA, Poland J, Ramsay L, Hedley $P E$, Waugh R. An evaluation of genotyping by sequencing (GBS) to map the Breviaristatum-e (ari-e) locus in cultivated barley. BMC Genomics. 2014;15(1): 104-15.

36. Carlson BM, Onusko SW, Gross JB. A high-density linkage map for Astyanaxmexicanus using genotyping-by-sequencing technology. G3. 2015; 5(2):241-51.

37. Woodward RW, Thieret JW. A genetic study of complementary genes for purple lemma, Palea and pericarp in barley (Hordeum vulgare L.). Agron J. 1953;45:182-5.

38. Franckowiak JD, Lundqvist U. New and revised descriptions of barley genes. Barley Genet Newsl. 1997;26:209.

39. Zhang MF, Jiang LM, Zhang DM, Jia GX. De novo transcriptome characterization of Lilium 'Sorbonne' and key enzymes related to the flavonoid biosynthesis. Mol Gen Genomics. 2015;290(1):399-412.

40. Larson RL, Bussard JB. Microsomal flavonoid 3'- monooxygenase from maize seedlings. Plant Physiol. 1986;80(2):483-6.

41. Halbwirth $\mathrm{H}$. The creation and physiological relevance of divergent hydroxylation patterns in the flavonoid pathway. Int J Mol Sci. 2010;11(2):595-621.

42. Li P, Li YJ, Zhang FJ, Zhang GZ, Jiang XY, Yu HM, Hou BK. The Arabidopsis UDP-glycosyl transferases UGT79B2 and UGT79B3, contribute to cold, salt and drought stress tolerance via modulating anthocyanin accumulation. Plant J. 2017:89(1):85-103.

43. Yonekura-Sakakibara K, Tanaka Y, Fukuchi-Mizutani M, Fujiwara H, Fukui Y, Ashikari T, Murakami Y, Yamaguchi M, Kusumi T. Molecular and biochemical characterization of a novel hydroxycinnamoyl-CoA: anthocyanin 3-O-glucoside6-O-acyltransferase from Perillafrutescens. Plant Cell Physiol. 2000;41(4):495-502.

44. Jiang F, Chen XP, Hu WS, Zheng SQ. Identification of differentially expressed genes implicated in peel color (red and green) of Dimocarpusconfinis. Springer Plus. 2016;5(1):1088-101.

45. Zhao L, Gao LP, Wang HX, Chen XT, Wang YS, Hua Y, Wei CL, Wan XC Xia T. The R2R3-MYB, bHLH, WD40, and related transcription factors in flavonoid biosynthesis. Funct Integr Genomics. 2013;13(1):75-98.

Ready to submit your research? Choose BMC and benefit from:

- fast, convenient online submission

- thorough peer review by experienced researchers in your field

- rapid publication on acceptance

- support for research data, including large and complex data types

- gold Open Access which fosters wider collaboration and increased citations

- maximum visibility for your research: over $100 \mathrm{M}$ website views per year

At BMC, research is always in progress.

Learn more biomedcentral.com/submissions 